\begin{tabular}{|c|c|}
\hline Title & Brownian motion in shear flow : Direct observation of anomal ous diffusion \\
\hline Author(s) & Orihara, Hiroshi; Takikawa, Yoshinori \\
\hline Citation & $\begin{array}{l}\text { Physical Review E, 84(6), } 061120 \\
\text { https://doi.org/10.1103/PhysRevE.84.061120 }\end{array}$ \\
\hline Issue Date & $2011-12$ \\
\hline Doc URL & http:/hdl.handle.net/2115/48132 \\
\hline Rights & @2011 A merican Physical Society \\
\hline Type & article \\
\hline File Information & PRE846_061120.pdf \\
\hline
\end{tabular}

Instructions for use 


\title{
Brownian motion in shear flow: Direct observation of anomalous diffusion
}

\author{
Hiroshi Orihara* and Yoshinori Takikawa \\ Division of Applied Physics, Faculty of Engineering, Hokkaido University, Sapporo, Hokkaido 060-8628, Japan
}

(Received 26 June 2011; revised manuscript received 15 September 2011; published 12 December 2011)

\begin{abstract}
Brownian motion in a simple shear flow has been experimentally investigated by using a different method for observation and analysis. A number of polystyrene spheres dispersed in sheared water were tracked with a confocal scanning laser microscope, and the time dependences of their coordinates were obtained. Since in the usual mean-square displacement in the flow direction the contribution from the Brownian motion is overwhelmed by that due to the convection, we considered an alternative displacement for which the convection effect could be removed. We found that the new mean-square displacement consists of the normal Einstein diffusion term, which is linear in $t$, and an anomalous $t^{3}$ term arising from the coupling between the diffusion along the velocity gradient and the convection.
\end{abstract}

DOI: 10.1103/PhysRevE.84.061120

PACS number(s): 05.40.Jc, 82.70.-y, 83.50.Ax

\section{INTRODUCTION}

Taylor dispersion is a well-known case in which the coupling between diffusion and convection plays an important role in a sheared fluid [1]. When a soluble substance is introduced into a fluid flowing slowly through a narrow tube, it spreads along the tube under the combined action of molecular diffusion and the variation of velocity over the cross section. Taylor verified, both experimentally and theoretically, that the spreading of the cross-sectionally averaged concentration followed an ordinary diffusion equation with an effective diffusion constant depending on the Poiseuille velocity profile and the molecular diffusion constant. For a Brownian particle undergoing shear, a similar effect is also expected. The Brownian dynamics has been theoretically studied on the basis of a convective diffusion equation [2-5], which was used by Taylor, and a Langevin equation [5-8]. Both give the same mean-square displacement (MSD) in the flow direction $x$ for a simple shear flow [4-7]:

$$
\left\langle(x(t)-x(0)-\dot{\gamma} z(0) t)^{2}\right\rangle=2 D t\left[1+\frac{1}{3}(\dot{\gamma} t)^{2}\right],
$$

where $\langle\cdots\rangle$ denotes the ensemble average, the $z$ axis is taken to be along the velocity gradient, and $D$ and $\dot{\gamma}$ are the diffusion constant and shear rate, respectively. Note that the displacement due to the shear flow along a stream line, $\dot{\gamma} z(0) t$, where it is assumed that the flow velocity is zero at $z=0$, is subtracted in the left-hand side of Eq. (1). The $t^{3}$ term in Eq. (1) is the most important characteristic of a free Brownian particle in simple shear flow, which becomes greater than the Einstein term $2 D t$ for large $\dot{\gamma} t$. The origin of the anomalous diffusion is as follows: the Brownian motion of a particle along the velocity gradient (the $z$ direction) causes a velocity change along the flow direction (the $x$ direction) and consequently a change in the displacement as well. Despite its importance and simple form, Eq. (1) has not been experimentally verified, although dynamic light scattering experiments related to this issue have been made [9-11]. The MSDs along $y$ (the vorticity direction) and $z$ obey the usual Einstein diffusion even in shear flow: $\left\langle[y(t)-y(0)]^{2}\right\rangle=\left\langle[z(t)-z(0)]^{2}\right\rangle=2 D t$.

\footnotetext{
*orihara@eng.hokudai.ac.jp
}

Recently, the coupling between diffusion and convection has been experimentally investigated through cross correlations of displacements along the $x$ and $z$ directions, $\langle x(t) z(0)\rangle$ and $\langle x(0) z(t)\rangle$, where a particle was trapped with an optical tweezer in a linear shear flow [12]. A strong time asymmetry was observed in the cross correlations as predicted theoretically [13]. This should disappear in the absence of flow, i.e., in the equilibrium state. Although the coupling was clearly shown, the $t^{3}$ anomaly for a free particle remains an open question. Experimentally, it may be difficult to trace a free particle in shear flow for a long enough time to calculate the MSD with sufficient statistical accuracy. Furthermore, the subtraction of the displacement due to flow as in Eq. (1) may be difficult because of the need to measure $z(0)$, although these can be done by simulations [14]. Here, it should be stressed that we can experimentally obtain $\left\langle[x(t)-x(0)]^{2}\right\rangle$ but this has a term $[\dot{\gamma} z(0) t]^{2}$ which would overwhelm the other two terms under available experimental conditions. In this paper, we describe how to overcome the above difficulties and experimentally verify the $t^{3}$ anomaly.

\section{EXPERIMENTAL AND ANALYSIS METHODS}

For tracing Brownian particles in shear flow, we used a system combining a cone-plate rheometer and a confocal scanning laser microscope (CSLM), as shown in Fig. 1 [15]. The diameter of the cone plate was $25 \mathrm{~mm}$. Observations of fluorescence beads dispersed in distilled water were made through the bottom glass plate of the rheometer with the CSLM [Fig. 1(a)]. The observations were taken $3 \mathrm{~mm}$ in from the edge of the rotating plate, where the gap was $170 \mu \mathrm{m}$. The focal plane of the CSLM was fixed at $10 \mu \mathrm{m}$ above the glass surface and two-dimensional images $(400 \mu \mathrm{m} \times 400 \mu \mathrm{m})$ were captured with a frame rate of $60 \mathrm{fps}$ for about $2 \mathrm{~min}$. We used a low numerical aperture $(\mathrm{NA})$ object lens $(20 \times$ and $\mathrm{NA}=0.50)$ so that we could observe particles in a layer with a thickness of about $10 \mu \mathrm{m}$ between $z_{1}=5 \mu \mathrm{m}$ and $z_{2}=15 \mu \mathrm{m}$ [Fig. 1(b)] by adjusting the threshold used for image thresholding. Under our experimental conditions, the diffusion length along the $z$ axis is smaller than $z_{1}$ so that the boundary effect can be neglected. All the particles in this layer were tracked and the time dependences of the $x$ and $y$ coordinates were obtained. 


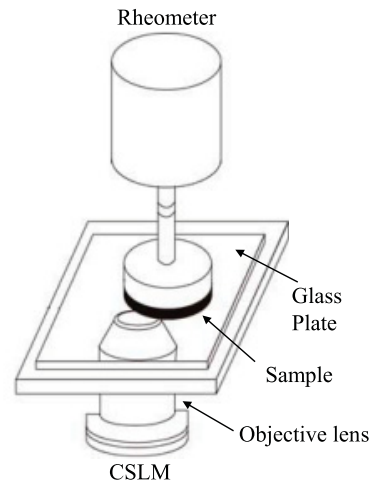

(a)

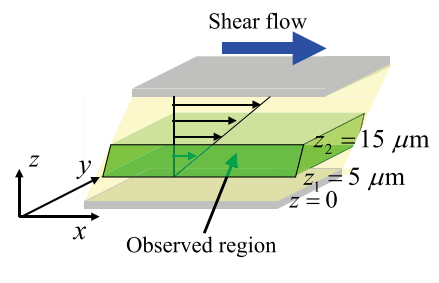

(b)
FIG. 1. (Color online) (a) Schematic illustration of system combining a rheometer and CSLM. (b) Details of the sample space. The observed region is a thin layer between $z=z_{1}(5 \mu \mathrm{m})$ and $z_{2}$ (15 $\mu \mathrm{m})$.

We had about 700 traces with a length greater than $2.5 \mathrm{~s}$ for one run. The data analyses were performed as follows.

We start with a Langevin equation for a particle subjected to simple shear flow [5]:

$$
\begin{aligned}
\zeta(\dot{x}-\dot{\gamma} z) & =R_{x}(t), \\
\zeta \dot{z} & =R_{z}(t),
\end{aligned}
$$

where $\dot{\gamma}$ is the shear rate, $\zeta=6 \pi a \eta$ with $a$ the particle radius and $\eta$ the shear viscosity, $R_{\alpha}$ is a random force, and the dots denote the time derivatives. The inertia terms have been dropped, as they are negligible in our experiment. The equation of motion for $y$ is similar to that for $z$. The random forces satisfy $\left\langle R_{\alpha}(t)\right\rangle=0$ and

$$
\left\langle R_{\alpha}(t) R_{\beta}\left(t^{\prime}\right)\right\rangle=2 \zeta k_{B} T \delta_{\alpha \beta} \delta\left(t-t^{\prime}\right) .
$$

The diffusion constant in Eq. (1) is given by $D=k_{B} T / \zeta$ (Einstein-Stokes relation). The solution of Eq. (2) can be written as

$$
\begin{aligned}
x(t) & =x(0)+\dot{\gamma} z(0) t+x_{B}(t), \\
z(t) & =z(0)+z_{B}(t),
\end{aligned}
$$

where $x(0)$ and $z(0)$ are the initial coordinates at $t=0$, and $x_{B}(t)$ and $z_{B}(t)$ are the stochastic parts, which satisfy Eq. (2) with initial conditions of $x_{B}(0)=z_{B}(0)=0$. The MSD of $x_{B}(t)$ is given by Eq. (1). As was mentioned above, it is experimentally difficult to obtain $x_{B}(t)$. Instead, we introduce a new variable:

$$
\begin{aligned}
\tilde{x}(t) & \equiv\{[x(2 t)-x(t)]-[x(t)-x(0)]\} / \sqrt{2} \\
& =\left[x_{B}(2 t)-2 x_{B}(t)\right] / \sqrt{2},
\end{aligned}
$$

where we have used Eq. (4a). Note that $\tilde{x}$ depends only on $x_{B}$, and is independent of either the initial position or the flow velocity. The MSD of $\widetilde{x}(t)$ can be calculated to be the same as that of $x_{B}(t)$, i.e., $\left\langle\widetilde{x}(t)^{2}\right\rangle=\left\langle x_{B}(t)^{2}\right\rangle$. This will be derived later. Furthermore, defining

$$
\begin{aligned}
\tilde{x}_{m, n}(t) \equiv & {[\{x(n t)-x[(n-1) t]\}} \\
& -\{x(m t)-x[(m-1) t]\}] / \sqrt{2},
\end{aligned}
$$

we can obtain

$$
\left\langle\widetilde{x}_{1,3}(t) \widetilde{x}_{2,4}(t)\right\rangle=\left\langle\widetilde{x}_{1,4}(t) \tilde{x}_{2,3}(t)\right\rangle=D t(\dot{\gamma} t)^{2},
$$

indicating that only the $t^{3}$ term can be extracted. In addition, $\left\langle\widetilde{x}_{1,2}(t) \tilde{x}_{3,4}(t)\right\rangle=0$.

When we calculated $\left\langle\widetilde{x}(t)^{2}\right\rangle$ and $\left\langle\widetilde{x}_{1,3}(t) \tilde{x}_{2,4}(t)\right\rangle$ from the experimentally obtained $x(t)$, we used the difference $\Delta x_{i j}(t) \equiv$ $\left[x_{i}(t)-x_{j}(t)\right] / \sqrt{2}$ for the $i$ th and $j$ th particles rather than $x(t)$ itself, because the captured images suffered small spatially uniform oscillations for some reason, which affected the results. It can be shown that both $x(t)$ and $\Delta x_{i j}(t)$ give the same averaged quantities unless there is any correlation between the $i$ th and $j$ th particles. We chose suitable pairs of particles, calculated $\left\langle\widetilde{x}(t)^{2}\right\rangle$ and $\left\langle\widetilde{x}_{1,3}(t) \tilde{x}_{2,4}(t)\right\rangle$ of the difference for each pair, and then averaged them over all the pairs. Furthermore, they were averaged over four runs to reduce statistical errors.

\section{RESULTS AND DISCUSSION}

In the measurements, fluorescence polystyrene spheres with diameters of 0.5 and $1 \mu \mathrm{m}$ (Fluospheres, Invitrogen) were used. They were dilutely dispersed in distilled water so that interactions between particles were negligible. Measurements were made at shear rates of 0,2 , and $4 \mathrm{~s}^{-1}$ at $25^{\circ} \mathrm{C}$. The MSD of $\tilde{x}(t)$ [ $\Delta x_{i j}(t)$ was actually used for calculation] at each shear rate is shown in Fig. 2. The MSD of $\tilde{y}(t)$, which is defined in a way similar to Eq. (5), is also shown. Also shown are the MSDs for a dispersion of $0.5 \mu \mathrm{m}$ particles in glycerine, which has almost no Brownian motion because of its large viscosity $(0.42 \mathrm{~Pa}$ s). These confirm the absence of extrinsic fluctuations. Without shear flow [Fig. 2(a)], $\left\langle\widetilde{x}(t)^{2}\right\rangle$ and $\left\langle\tilde{y}(t)^{2}\right\rangle$ are the same for each particle diameter, and they are proportional to time. The slopes give diffusion constants for 0.5 and $1 \mu \mathrm{m}$ particles as 0.91 and $0.45 \mu \mathrm{m}^{2} \mathrm{~s}^{-1}$, respectively, which are in good agreement with those calculated from the Einstein-Stokes relation, 0.94 and $0.47 \mu \mathrm{m}^{2} \mathrm{~s}^{-1}$.

In the presence of shear flows, $\left\langle\widetilde{x}(t)^{2}\right\rangle$ deviates from $\left\langle\widetilde{y}(t)^{2}\right\rangle$, which is independent of shear rate. They coincide at initial times for each particle, while $\left\langle\widetilde{x}(t)^{2}\right\rangle$ increases more than $\left\langle\tilde{y}(t)^{2}\right\rangle$ at later times, indicating the existence of the anomalous diffusion. As can be seen from Figs. 2(b) and 2(c), the anomalous diffusion increases as the shear rate is raised, which coincides with the prediction of Eq. (1). In the inset of Fig. 2(c), we show a log-log plot of $\left\langle\tilde{x}(t)^{2}\right\rangle-\left\langle\tilde{y}(t)^{2}\right\rangle$, which should be equal to $2 / 3 D t(\dot{\gamma} t)^{2}$. The exponents for the 0.5 and $1 \mu \mathrm{m}$ particles are, respectively, $2.90 \pm 0.01$ and $2.96 \pm 0.01$, clearly indicating the $t^{3}$ dependence. The solid lines in Figs. 2(b) and 2(c) denote theoretical curves calculated from Eq. (1) with $D$ experimentally obtained from the slopes of $\left\langle\widetilde{x}(t)^{2}\right\rangle$ in Fig. 2(a), clearly verifying the equation, although the theoretical ones are slightly larger than the experimental ones for all the MSDs of $\left\langle\tilde{x}(t)^{2}\right\rangle$. This discrepancy will be discussed later. Here, we would like to emphasize that the usual $\left\langle[x(t)-x(0)]^{2}\right\rangle$ is very large due to the contribution from the flow; for example, about $2000 \mu \mathrm{m}^{2}$ at $t=1 \mathrm{~s}$ and $\dot{\gamma}=4 \mathrm{~s}^{-1}$, which is much larger than the corresponding value of $\left\langle\widetilde{x}(t)^{2}\right\rangle, 10.3 \mu \mathrm{m}^{2}$. This demonstrates the usefulness of our method. Figure 3 shows $\left\langle\widetilde{x}_{1,3}(t) \widetilde{x}_{2,4}(t)\right\rangle$ at shear rates of 0 , 2 , and $4 \mathrm{~s}^{-1}$. Since $\left\langle\widetilde{x}_{1,3}(t) \tilde{x}_{2,4}(t)\right\rangle$ has no contribution from normal diffusion, it is almost zero without shear flow, as shown 


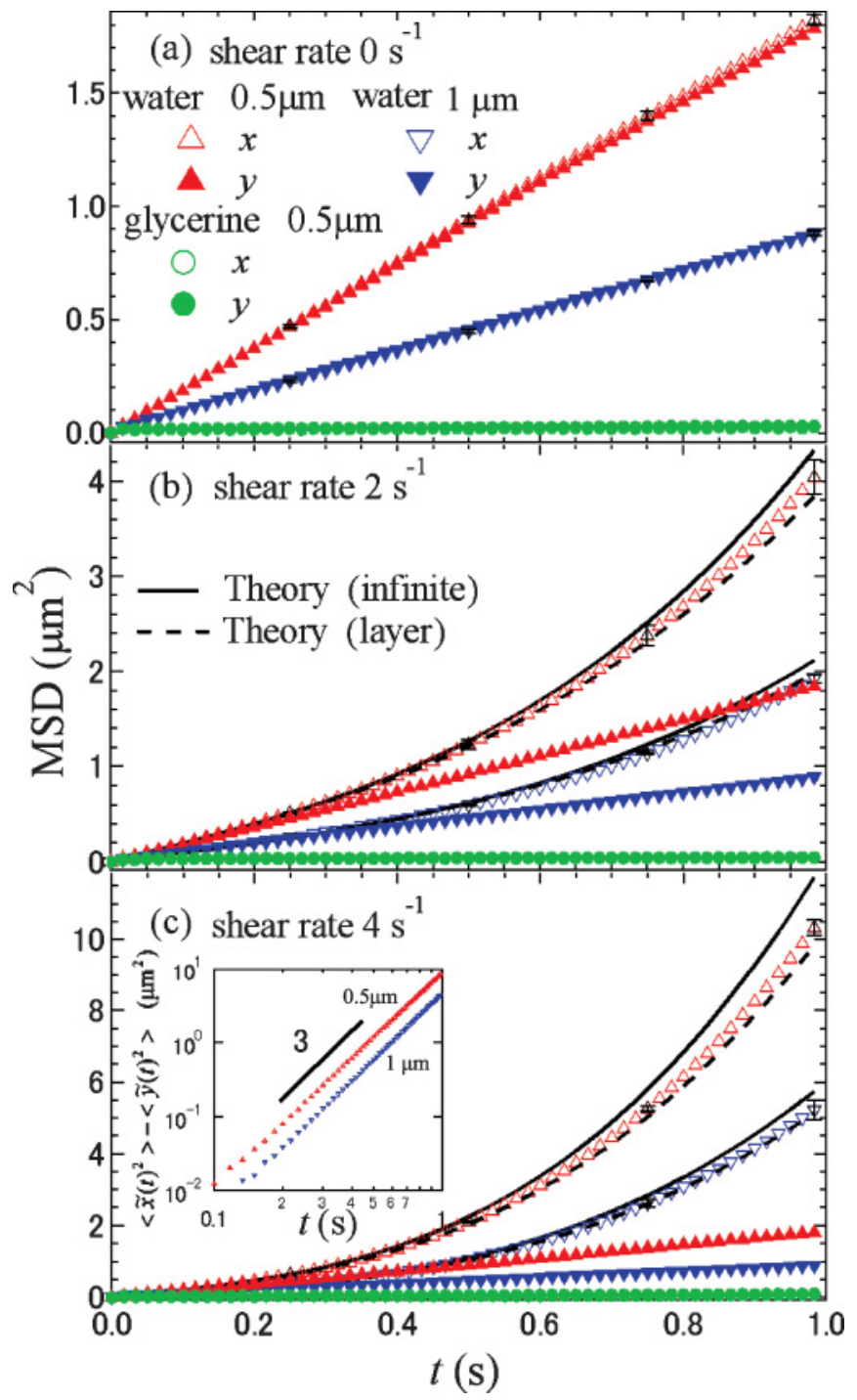

FIG. 2. (Color online) MSDs of $\widetilde{x}(t)$ and $\tilde{y}(t)$ for particles with diameters 0.5 and $1 \mu \mathrm{m}$ in distilled water at shear rates of (a) 0 , (b) 2 , and (c) $4 \mathrm{~s}^{-1}$ at $25^{\circ} \mathrm{C}$. For reference, results for particles with a diameter of $0.5 \mu \mathrm{m}$ in glycerine are also shown. Solid and dashed curves are theoretical results for an infinite system Eq. (1) and a layer Eq. (10), respectively. A $\log -\log$ plot of $\left\langle\widetilde{x}(t)^{2}\right\rangle-\left\langle\widetilde{y}(t)^{2}\right\rangle$ is also shown for 0.5 and $1 \mu \mathrm{m}$ particles in the inset of (c).

in Fig. 3(a). In the presence of shear flows [Figs. 3(b) and 3(c)], in contrast, the anomalous diffusion appears and increases with the shear rate. The exponent of the anomaly is almost 3 , as is seen from the insets in Fig. 3. However, theoretical values (solid lines) calculated from Eq. (7) are larger than the experimental values. This tendency is more remarkable than in $\left\langle\widetilde{x}(t)^{2}\right\rangle$. The discrepancies between experiment and theory are ascribable to the fact that the observed region was confined to a thin layer of about $10 \mu \mathrm{m}$ in our experiment, while the theoretical result in Eq. (1) was obtained for an infinite system.

Before discussing the discrepancies, we show the time evolution of the density distribution of $0.5 \mu \mathrm{m}$ particles at $\dot{\gamma}=4 \mathrm{~s}^{-1}$. In Fig. 4, [ $\left.\tilde{x}(t), \tilde{y}(t)\right]$ is plotted for 1300 traces at $t=$ (a) 0.2 , (b) 0.4 , (c) 0.6 , and (d) $0.8 \mathrm{~s}$. The appearance

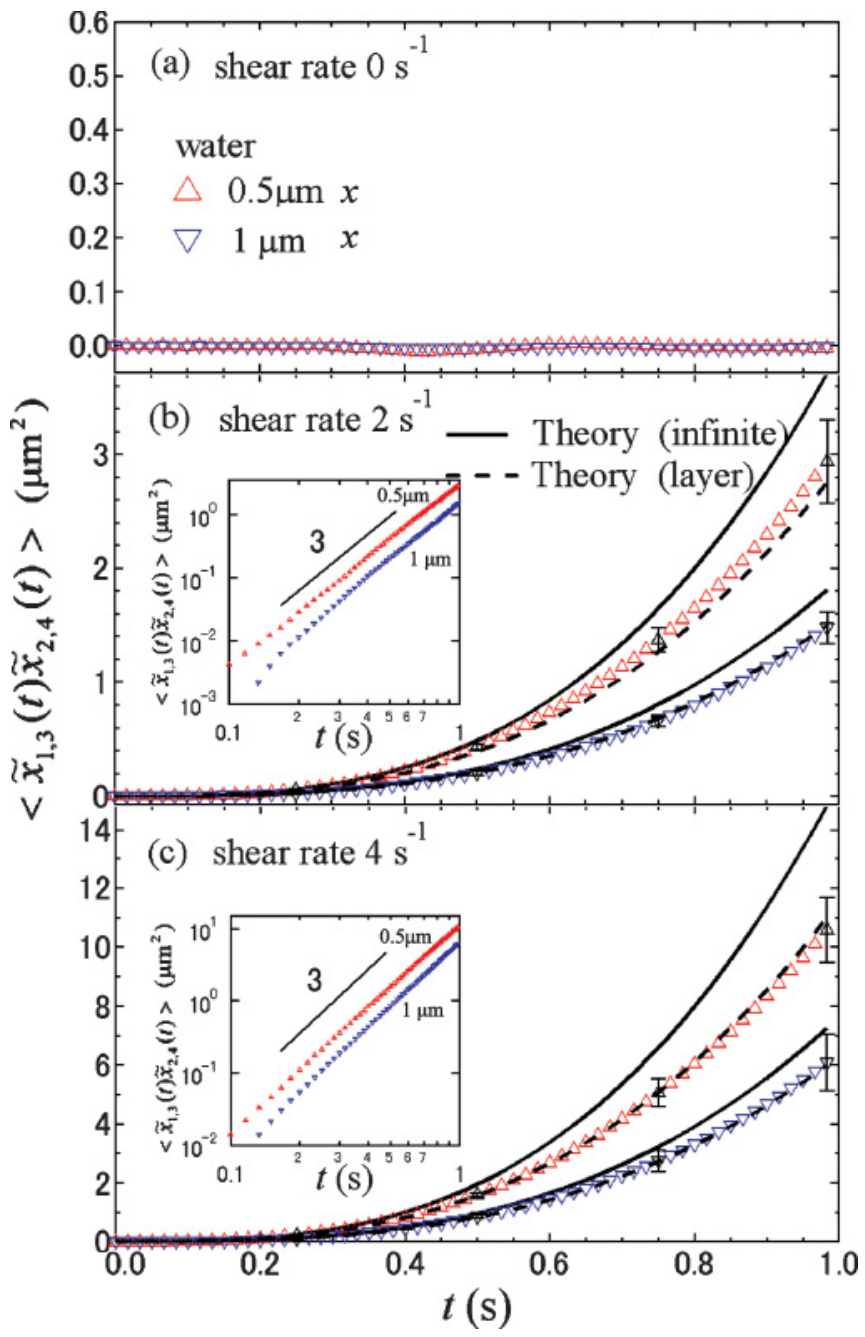

FIG. 3. (Color online) $\left\langle\widetilde{x}_{1,3}(t) \widetilde{x}_{2,4}(t)\right\rangle$ for particles with diameters 0.5 and $1 \mu \mathrm{m}$ in distilled water at shear rates of (a) 0 , (b) 2 , and (c) $4 \mathrm{~s}^{-1}$ at $25^{\circ} \mathrm{C}$. Log-log plots are also shown in the insets of (b) and (c). Solid and dashed curves are theoretical results for an infinite system Eq. (7) and a layer Eq. (12), respectively.

of anisotropy due to the anomalous diffusion is clearly seen at later times. The normalized two-dimensional distribution function projected onto the $x-y$ plane is given by [2-5]

$$
\begin{aligned}
\rho(x, y, t) \equiv & \frac{1}{2 \pi \sqrt{\left\langle x_{B}(t)^{2}\right\rangle\left\langle y_{B}(t)^{2}\right\rangle}} \exp \left[-x^{2} / 2\left\langle x_{B}(t)^{2}\right\rangle\right. \\
& \left.-y^{2} / 2\left\langle y_{B}(t)^{2}\right\rangle\right] .
\end{aligned}
$$

Now, let us consider the effect of the finiteness of the observed region. From Eq. $(2 \mathrm{a}),\left\langle x_{B}(t)^{2}\right\rangle$ is given by

$$
\begin{aligned}
\left\langle x_{B}(t)^{2}\right\rangle= & \zeta^{-2} \int_{0}^{t} d \tau_{1} \int_{0}^{t} d \tau_{2}\left\langle R_{x}\left(\tau_{1}\right) R_{x}\left(\tau_{2}\right)\right\rangle \\
& +\dot{\gamma}^{2} \int_{0}^{t} d \tau_{1} \int_{0}^{t} d \tau_{2}\left\langle z_{B}\left(\tau_{1}\right) z_{B}\left(\tau_{2}\right)\right\rangle .
\end{aligned}
$$

With the use of Eq. (3), the first term on the right-hand side becomes $2 D t$. But, the second term does not coincide with the $t^{3}$ term in Eq. (1) when the observed region is confined to a thin layer with the layer normal being along the $z$ direction, because 

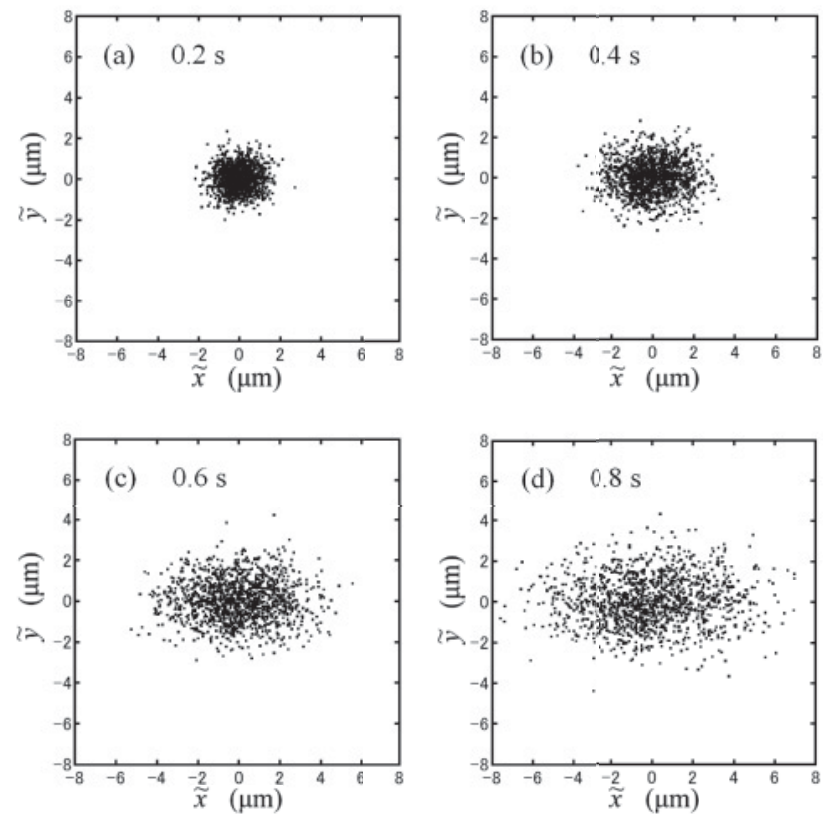

FIG. 4. Time evolution of the particle density for $\dot{\gamma}=4 \mathrm{~s}^{-1}$ : plots of $[\tilde{x}(t), \tilde{y}(t)]$ for 1300 traces at $t=$ (a) 0.2 , (b) 0.4 , (c) 0.6 , and (d) $0.8 \mathrm{~s}$.

the range of $z_{B}$ is finite and so $\left\langle z_{B}\left(\tau_{1}\right) z_{B}\left(\tau_{2}\right)\right\rangle$ is bounded above. This indicates that $\left\langle x_{B}(t)^{2}\right\rangle$ for a thin layer should always be smaller than that for an infinite observed region. Note that the decrease of $\left\langle x_{B}(t)^{2}\right\rangle$ is due to the finiteness of the observed region, but not to the boundary effect of the top or bottom plate. In our analysis, we used $\left\langle\widetilde{x}(t)^{2}\right\rangle$, which is calculated as

$$
\left\langle\widetilde{x}(t)^{2}\right\rangle=2 D t+\dot{\gamma}^{2} \int_{0}^{2 t}\left[I_{z}(\tau)-I_{z}(t)\right] d \tau,
$$

with

$$
I_{z}(t) \equiv \int\left\langle z_{B}(t)^{2}\right\rangle d t
$$

where we have used the relation $\left\langle z_{B}\left(t_{1}\right) z_{B}\left(t_{2}\right)\right\rangle=$ $\left\langle z_{B}\left[\min \left(t_{1}, t_{2}\right)\right]^{2}\right\rangle$, derived from Eqs. (2b) and (3). It is readily confirmed that substitution of $I_{z}(t)=D t^{2}$ for an infinite observed region into Eq. (10) yields Eq. (1). Similarly, we obtain

$$
\begin{aligned}
\left\langle\widetilde{x}_{1,3}(t) \widetilde{x}_{2,4}(t)\right\rangle & =\left\langle\widetilde{x}_{1,4}(t) \widetilde{x}_{2,3}(t)\right\rangle \\
& =\frac{1}{2} \dot{\gamma} t\left[I_{z}(3 t)-2 I_{z}(2 t)+I_{z}(t)\right] .
\end{aligned}
$$

We can estimate $\left\langle z_{B}(t)^{2}\right\rangle$ for a thin layer. From Eq. (4), $\left\langle z_{B}(t)^{2}\right\rangle$ can be rewritten as $\left\langle[z(t)-z(0)]^{2}\right\rangle$, indicating that the probability density functions (PDFs) for both $z(0)$ and $z(t)$ are needed. In the present experiment, particles enter the layer mainly through the plane perpendicular to the $x$ axis, and the flux is proportional to the flow velocity. Therefore, the PDF for $z(0)$ may be proportional to $\dot{\gamma} z(0)$ if particles entering the layer through the upper and lower surfaces are negligible, which is true when the shear rate is large or the diffusion is small. Thus, by taking into account that the particle density is constant independent of the position, the normalized PDF for $z(0)$ becomes $2 z(0) /\left(z_{2}^{2}-z_{1}^{2}\right)$, where $z_{2}$ and $z_{1}$ are the positions of the upper and lower boundaries. Note that the PDF is independent of the shear rate. On the other hand, the diffusion in the $z$ direction is normal and, therefore, the probability density for a particle obeys the usual diffusion equation, the solution of which is a Gaussian function with a variance of $2 D t$. Therefore, we can approximate $\left\langle z_{B}(t)^{2}\right\rangle$ as

$$
\left\langle z_{B}(t)^{2}\right\rangle \simeq \frac{2}{z_{2}^{2}-z_{1}^{2}} \int_{z_{1}}^{z_{2}} z_{0} d z_{0} \frac{\int_{z_{1}}^{z_{2}}\left(z-z_{0}\right)^{2} f\left(z-z_{0}, t\right) d z}{\int_{z_{1}}^{z_{2}} f\left(z-z_{0}, t\right) d z},
$$

with

$$
f(z, t) \equiv \frac{1}{\sqrt{8 \pi D t}} \exp \left(-z^{2} / 4 D t\right),
$$

where we have introduced $\int_{z_{1}}^{z_{2}} f\left(z-z_{0}, t\right) d z$ in the denominator to make the particle density constant in the layer. This may compensate for the absence of diffusion of particles into the layer through the upper and lower boundaries. Theoretical curves are shown by dashed lines in Figs. 2 and 3. They are in satisfactory agreement with the experimentally obtained data.

\section{CONCLUSIONS}

We have presented a different method to obtain MSDs of a free particle in shear flow and have verified the anomalous diffusion, which is caused by the coupling between the normal diffusion along the velocity gradient and the convection. We also investigated the effect of the finiteness of the observed region, which makes MSDs smaller than that for the infinite system. A good agreement was obtained between experiment and theory by taking into account the finite effect. An interesting challenge for the future may be the application of this method to dense suspensions, in which the motion of the particles due to shear-induced interparticle interactions is greater than that arising from Brownian motion [16].

\section{ACKNOWLEDGMENTS}

This work was partially supported by Grant-in-Aid for Scientific Research [Priority Area "Soft Matter Physics" (Contract No. 463) and C (Contract No. 22540416)] from the Ministry of Education, Culture, Sports, Science and Technology of Japan.
[1] G. Taylor, Proc. R. Soc. London, Ser. A 219, 186 (1953).

[2] D. E. Elrick, Aust. J. Phys. 15, 283 (1962).

[3] A. Okubo, J. Oceanogr. Soc. Jpn. 24, 20 (1968).

[4] T. G. M. van de Ven, J. Colloid Interface Sci. 62, 352 (1977).
[5] R. T. Foister and T. G. M. van de Ven, J. Fluid Mech. 96, 105 (1980).

[6] C. van den Broeck, J. M. Sancho, and M. S. Miguel, Physica A 116, 448 (1982). 
[7] P. Schram and S. A. Trigger, Physica B 228, 91 (1996).

[8] Y. Katayama and R. Terauti, Eur. J. Phys. 17, 136 (1996).

[9] B. J. Ackerson and N. A. Clark, J. Phys. 42, 929 (1981).

[10] G. G. Fuller, J. M. Rallison, R. L. Schmidt, and L. G. Leal, J. Fluid Mech. 100, 555 (1980).

[11] M. Hoppenbrouwers and W. van de Water, Phys. Fluids 10, 2128 (1998).
[12] A. Ziehl, J. Bammert, L. Holzer, C. Wagner, and W. Zimmermann, Phys. Rev. Lett. 103, 230602 (2009).

[13] L. Holzer, J. Bammert, R. Rzehak, and W. Zimmermann, Phys. Rev. E 81, 041124 (2010).

[14] T. Iwashita and R. Yamamoto, Phys. Rev. E 79, 031401 (2009).

[15] H. Orihara, Y. Nishimoto, K. Aida, and Y. H. Na, Phys. Rev. E 83, 026302 (2011).

[16] D. Leighton and A. Acrivos, J. Fluid Mech. 177, 109 (1987). 